\title{
Translation, Cultural Adaptation and Validation to Brazilian Portuguese of the Tinnitus Functional Index Questionnaire
}

\author{
${ }^{1}$ Speech Therapy Department, Universidade Federal da Paraíba, João \\ Pessoa, PB, Brazil \\ ${ }^{2}$ Associate Program, Universidade Estadual de Londrina e \\ Universidade Norte do Paraná, Londrina, PR, Brazil \\ ${ }^{3}$ Speech Therapy Department, Universidade Federal de São Paulo, \\ São Paulo, SP, Brazil \\ ${ }^{4}$ Otorrhinolaryngology Postgraduate Program, Faculty of Medicine, \\ Universidade de São Paulo, São Paulo, SP, Brazil \\ ${ }^{5}$ Tinnitus Research Group, Hospital das Clínicas da Faculdade de \\ Medicina da Universidade de São Paulo, São Paulo, SP, Brazil \\ ${ }^{6}$ Speech Therapy Department, Unicesumar, Maringá, PR, Brazil \\ Int Arch Otorhinolaryngol 2022;26(3):e304-e309.
}

Marine Raquel Diniz da Rosa ${ }^{10}$ Marcelo Yugi Doi ${ }^{20}$ Fátima Cristina Alves Branco-Barreiro ${ }^{30}$

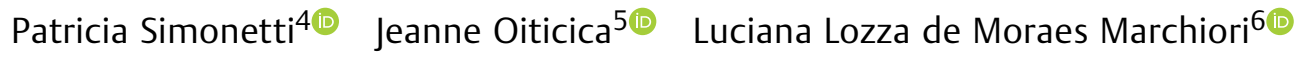

\begin{abstract}
Address for correspondence Marcelo Yugi Doi, PhD, Fonoaudiologia, Universidade Estadual de Londrina e Universidade Norte do Paraná (UEL-UNOPAR), Londrina, PR, 86041-120, Brazil (e-mail: marcelo.doi@unopar.br; marcelodoi21@gmail.com).
\end{abstract}

\begin{abstract}
Keywords

- tinnitus

- questionnaires

- validation studies

Introduction Tinnitus affects a large portion of the world's population. There are several questionnaires being used for the evaluation of the severity of tinnitus and its impact in quality of life; however, they do not measure treatment-related changes. So, a new self-reported questionnaire was developed, the Tinnitus Functional Index (TFI), which has been translated into several languages.

Objective To perform the translation, cultural adaptation and validation of the TFI questionnaire for Brazilian Portuguese.

Method This is a multicenter project divided into two stages: translation and cultural adaptation; and validation and reliability. For the validation, the Brazilian Portuguese version of the TFI was correlated with the Tinnitus Handicap Inventory (THI) domains for tinnitus and quality of life and was evaluated by the Spearman $\rho$ test. The reliability and internal consistency were evaluated by the Cronbach $\alpha$ test.

Result The Brazilian version of the TFI was obtained through an initial translation process, synthesis of translations, backtranslation and evaluation by a committee of experts. This version was then applied in 88 patients complaining of tinnitus from speech therapy and otorhinolaryngology outpatient clinics of the three school clinics. The Brazilian version presented high reliability, as evidenced by the Cronbach $\alpha$ value ( $\alpha$ $=0.870$ ), and strong correlation (rho $=0.760$ and $p=0.000$ ).

Conclusion The high reliability found in the results demonstrates that the Brazilian Portuguese version of the TFI is a valid and reliable instrument to evaluate the severity and impact of tinnitus on quality of life and changes related to its treatment.
\end{abstract}

received

December 20, 2019

accepted after revision

February 14, 2021

published online

August 13, 2021
DOI https://doi.org/ $10.1055 / \mathrm{s}-0041-1730347$. ISSN 1809-9777.

\footnotetext{
(c) 2021. Fundação Otorrinolaringologia. All rights reserved.

This is an open access article published by Thieme under the terms of the Creative Commons Attribution-NonDerivative-NonCommercial-License, permitting copying and reproduction so long as the original work is given appropriate credit. Contents may not be used for commercial purposes, or adapted, remixed, transformed or built upon. (https://creativecommons.org/ licenses/by-nc-nd/4.0/) Thieme Revinter Publicações Ltda., Rua do Matoso 170, Rio de Janeiro, RJ, CEP 20270-135, Brazil
} 


\section{Introduction}

Tinnitus is a common, persistent symptom characterized by a sound sensation in the "ears" or in the "head" that is not produced by mechanical-acoustic signals from the external environment. It can sound like noise but is more often a subjective perception that people are not able to describe objectively, only grade it on a scale ranging from low to high. ${ }^{1}$ A recent study showed the prevalence of the symptom in $22 \%$ of the individuals in the city of São Paulo, state of São Paulo, Brazil. $^{2}$

Tinnitus severity is variable and is often evaluated by measuring its functional impact on quality of life. Some factors seem to influence the perception of tinnitus of the patient. The association of tinnitus with anxiety, insomnia, depression, and other psychiatric comorbidities ${ }^{3}$ has been shown in the literature as important factors contributing to tinnitus severity. ${ }^{4,5}$ Cognitive processing speed has also been pointed out as a factor that can predict the severity of the symptom. ${ }^{6}$ Moreover, the presence of hearing loss and hyperacusis associated with tinnitus seems to increase the perception of the impact of the symptom. ${ }^{3,7}$

Tinnitus can be perceived as a whistle, a whisper, a buzzing, and so forth. It can be either single-toned or multitoned, which makes it even more difficult to be evaluated. Tinnitus can be perceived in one or both ears, in the head, or in no specific location. ${ }^{8}$

Studies $^{9,10}$ show that the psychoacoustic characteristics of tinnitus, such as pitch and loudness, do not explain the discomfort caused by it. Psychoacoustic measurements used to estimate those characteristics are seemingly unrelated to the impact of tinnitus, as assessed by a subjective outcome instrument. These psychoacoustic measurements do not assess the reactions to tinnitus. ${ }^{10}$

The heterogeneity of the clinical aspects of tinnitus and its pathophysiological mechanisms makes the evaluation and diagnosis of the symptom a difficult task, which has been reflected in treatment outcomes and in the variety of responses among individuals. ${ }^{11}$ Thus, there is a need for evaluative methods that best characterize tinnitus, regarding both the characteristics of the symptom itself and the comorbidities involved. ${ }^{12}$

The health status of the population is evaluated with laboratory and functional tests, clinical and personal knowledge evaluations, and their beliefs, covering both the characteristics and comorbidities of tinnitus. ${ }^{13}$ Self-assessment questionnaires have been used as indicators for monitoring the health status of the population according to its various domains of tinnitus impact. ${ }^{14}$

When it comes to tinnitus and the difficulty of objective evaluative methods, some questionnaires have been used as a tool to evaluate the degree of discomfort/severity of the symptom. Some of the most commonly used in the literature are the Tinnitus Handicap Inventory (THI); the Tinnitus Severity Scale (TSS); the Tinnitus Handicap Questionnaire (THQ); the Subjective Tinnitus Severity Scale (STSS); the Tinnitus Reaction Questionnaire (TRQ); the Tinnitus Ques- tionnaire (TQ); the Tinnitus Severity Index (TSI); the Tinnitus Severity Questionnaire (TSQ); the Tinnitus Catastrophizing Scale (TCS); the Fear of Tinnitus Scale (FTS); the Tinnitus Vigilance Awareness Questionnaire (TVAQ); the Tinnitus Primary Function Questionnaire (TPFQ); and the Tinnitus Functional Index (TFI).

Of the instruments used to evaluate the self-perception tinnitus severity and symptom improvement in different phases of the treatment, the THI questionnaire is the only one that has been translated and adapted into Portuguese. ${ }^{15}$ The THI is the most used questionnaire because it is easy to apply and has already been translated and validated in several languages. ${ }^{16-18}$ However, the THI was not created to respond accurately to treatment-related changes. For a treatment to be considered effective, the difference between pre- and post-intervention scores would have to be of at least 20 points. $^{19}$

Both the TFI and the THI can be used to assess the severity and the negative impact of tinnitus and to measure changes related to tinnitus treatment (response). The TFI covers different domains of tinnitus severity (eight subscales), which allows for significant clinical trials. ${ }^{20,21}$ The advantage of the TFI is that it was built, among other things, to accurately respond to treatment-related changes for tinnitus research and clinical practice. ${ }^{22}$

The TFI was developed over 4 years in the United States to provide a tinnitus severity scale, identifying with its domains its impact on symptom severity, as well as a sensitive measure of severity change. With exploratory factor analysis, tinnitus domains were defined in eight different subscales (factors): intrusion, sense of control, cognition, sleep, auditory, relaxation, quality of life, and emotional distress. ${ }^{23-25}$ In the original version, the Cronbach $\alpha$ test presents high internal consistency $(p=0.80)$ and reliability (intraclass correlation coefficient [ICC]: 0.91), and its construct validity was demonstrated with a strong correlation with the THI $(r=0.82)$ and the THQ $(r=0.82){ }^{25}$ It also showed moderate correlations with the Beck Depression Inventory-Primary Care and demonstrated good divergent validity. ${ }^{21}$

Since 2012, the TFI has been translated into various languages, and was validated in different cultures. The Dutch version is suitable for measuring in clinical or research settings. It has internal consistency $(\alpha=0.96)$ and statistically significant correlations with the visual analogue scale (VAS) for maximum loudness $(r=0.59$; $p<0.001)$, VAS for mean loudness $(r=0.66 ; p<0.001)$, and percentage of time aware of tinnitus $(r=0.58$; $p<0.001$ ) in 263 patients. $^{22}$

British research assessed the relevant psychometric TFI properties of volunteers, specifically the factor structure, reproducibility, validity, and responsiveness of the questionnaire, guided by quality criteria for the measurement properties of health-related questionnaires. The participants completed up to eight commonly used assessment questionnaires, including the TFI, the THI, the THQ a VAS of loudness (VAS-Loudness), Percentage Annoyance question, the Beck Depression Inventory (BDI), the Beck Anxiety 
Inventory (BAI), and the World Health Organization Quality of Life-Bref (WHOQOL-BREF). A series of analyses assessed the study objectives. Forty-four participants completed the TFI in a second visit (within 7 to 21 days and before receiving any intervention), providing data for reproducibility assessments. Even though the proposed eight-factor structure was not fully confirmed for this population, the TFI appears to cover multiple symptom domains and to measure the construct of tinnitus with excellent reliability in distinguishing between patients. While the TFI may distinguish those whose tinnitus is not a problem, floor effects in many items mean it is less appropriate as a measure of the change in this subgroup. Further investigation is needed to determine whether these effects are relevant in other populations. ${ }^{25}$

The translated and validated Polish version of the TFI has high internal consistency ( $\alpha=0.96$ ), and the reliability of its different subscales range from 0.82 to $0.95 .^{26}$ The Swedish version of the TFI (TFI-SE) was developed and had its validity and reliability investigated in 100 tinnitus patients. The results showed good internal consistency $(\alpha=0.95)$ and high test-retest reliability (ICC $=0.93)$. It also supported the eight-factor structure proposed for the original TFI, and there was a high correlation between the TFI-SE and the THI $(r=0.8 ; p<0.01)$ and a low correlation between the TFI-SE and the Hospital Anxiety and Depression Scale (HADS-D) $(r=0.60 ; p<0.01)$ and HADS$\mathrm{A}(\mathrm{r}=0.59 ; p<0.01){ }^{27}$

A German version of the TFI was also validated for Switzerland and showed an excellent internal consistency (0.97) and an excellent test-retest reliability of 0.91 . The convergent validity with the THI was high $(r=0.86)$. The discriminant validity with the BAI and the BDI showed moderate results (BAI: $r=0.60$; and $\mathrm{BDI}: \mathrm{r}=0.65$ ). ${ }^{28}$ In 2018, a psychometric evaluation of the TFI was undertaken on a sample of 318 patients in New Zealand. The results showed Cronbach $\alpha$ and ICC of 0.7 for the overall TFI and each of its subscales, indicating high internal consistency and testretest reliability. The clinical trial evaluation showed good test-retest reliability (a separate sample of 40 research volunteers) and agreement between no-treatment baselines with the smallest detectable change of 4.8 points. ${ }^{23}$

The Chinese version of the TFI was conducted with 124 adults in Hong Kong. The results showed internal consistency reliability $(\alpha=0.94)$ and test-retest reliability $(I C C=0.84)$. The TFI-CH has eight factors, which are precisely the same as the original version, and a strong correlation of the overall scale with other tinnitus-related distress measures $(\mathrm{r}=0.86$; $p<0.01)^{26}$

However, there is no Brazilian Portuguese version yet. Questionnaires are essential for measuring tinnitus severity and intervention-related change, but there is no standard instrument routinely used in research settings. Most tinnitus questionnaires are optimized for measuring severity, but not change. However, the TFI claims to be optimized both to measure severity and to respond to treatment-related changes.
Considering the high number of patients complaining of tinnitus and the existence of only one questionnaire in Portuguese for the evaluation of tinnitus severity and follow-up purposes, there is a need for the translation, adaptation, and validation of other instruments, such as the TFI.

The cultural adaptation of an evaluation instrument is of great importance, since the plain literal translation of an instrument does not consider the cultural and social differences that may exist in relation to the original tool. The instrument must be first culturally adapted and applied to determine the semantic and cultural equivalence of the evaluated items. $^{29}$

Therefore, the objective of the present study was to translate the TFI into Brazilian Portuguese, validate it, and perform a cultural adaptation of the questionnaire.

\section{Method}

This is a cross-sectional study with the translation, crosscultural adaptation, and validation of the TFI for its evaluation.

\section{Participants}

A multicenter study was conducted jointly by the postgraduate program (MSc) in Neurosciences and Behavior of the University Federal da Paraiba (UFPB, in the Portuguese acronym), the associated Ph.D. program in Science Rehabilitation of the Universidade Estadual de Londrina e Universidade Norte do Paraná (UEL/UNOPAR, in the Portuguese acronym), and the Hospital das Clínicas da Faculdade de Medicina da Universidade de São Paulo. The present study was approved by the Research Ethics Committee under CAAE 55153316.1.1001.5188.

A total of 88 individuals complaining of tinnitus participated in the study: 22 patients from the Northeast Region of Brazil, 32 from a university of the South Region, and 34 from the Tinnitus Research Group in the Southeast Region. Their age ranged from 20 to 88 years old (mean age, 56 years old); 51 patients were female and 37 were males.

\section{Procedures}

First, the author of the questionnaire was contacted to request their consent to use it. Subsequently, a license agreement was signed (Annex I).

The study was conducted in two steps: translation and cultural adaptation; validation and reliability.

Step 1 - Translation and cultural adaptation: the procedures performed at this step were based on Guillemin et al. $^{28}$

1. Stage 1 (initial translation): The TFI was translated into Portuguese by two professionals with tinnitus expertise, fluent in the original language of the instrument. They did not know each other, neither were acquainted with the material - one was aware of the objectives of the translation, while the other was neither aware nor 
informed. This procedure was followed to generate two independent documents: $\mathrm{T} 1$ and $\mathrm{T} 2$.

2. Stage 2 (synthesis of the translations): The two resulting documents (T1 and T2) were analyzed by a reviewer group of Brazilian professionals fluent in English from related areas (otorhinolaryngologists and audiologists). This group reduced the differences found in the translations by consensus, choosing the best expressions and words in all questions, and adapting the text to Brazilian cultural knowledge. From there, the T12 version was developed as a single document.

3. Stage 3 (back-translation [BT]): The T12 version was translated back into the original language (English) by two native English translators, without knowing the objectives of the work. These translators were not allowed to get in touch with the original English version to avoid any possible influence on the translation of words. After that, the BT was sent to the original author, who approved it.

4. Stage 4 (committee of experts): Two otorhinolaryngologists, three audiologists and speech therapists, one physical therapist, and one translator participated in this stage. The health professionals were also professors in masters' programs and, therefore, were familiar with scientific methodologies. This committee compared the T1, T2, T12, and BT versions with the original English version, verifying the semantic, idiomatic, cultural, and conceptual equivalences. In the end, the committee developed a version that was submitted to the pretest (BT12).

5. Stage 5 (pretest): The prefinal version (BT12) was thus submitted to pretest in patients who participated in a tinnitus research project from the Integrated Audiology and Speech Therapy Clinic of the UNOPAR and the UFPB. The participants were informed about the objective of the instrument and were instructed to evaluate it regarding the time of completion, clarity, and understandability. They were also asked to give their critical feedback and suggestions on the content and makeup of the instrument. The doubts that came up during the application of the questionnaire were answered, and the questions were verbally explained. Subsequently, the semantic agreement between the original instrument and the Portuguese version was evaluated. Thus, the pretest phase was completed, and the version was approved to be used in the final test to evaluate psychometric measurements.

Step 2 - Evaluation of psychometric properties (validity and reliability)

A total of 89 volunteers, whose mean age was 56 years old and who had chronic tinnitus (6 months) participated in this stage.

The volunteers, all of whom signed the Informed Consent Form, were recruited from the three teaching clinics participating in the study.

The final version of the TFI was applied in separate room interviews, and the patients answered 25 questions subdi- vided into 8 subscales (persistence, sense of control, cognition, sleep, hearing, relaxation, quality of life, and emotional). For each question, the patient should mark a scale from 0 to 10 , in which zero corresponded to less discomfort, difficulty, or tinnitus interference in their life, and the closer to 10 , the greater the discomfort, difficulty, or interference. After application, the answers were tabulated in scores.

The reliability was studied by evaluating internal consistency with Cronbach $\alpha$ statistic. The expected Cronbach $\alpha$ value for the study of a scale was $\geq 0.7$.

The THI-Brazilian version was applied to the criterion validity analysis.

The THI was administered in an interview, and the volunteer chose 1 of the 3 possible answers to each of the 25 questions: "yes" (4 points), "no" (0 points), or "sometimes" (2 points). Each question relates to one of the domains: functional, emotional, or catastrophic. Thus, the sum of the obtained scores could range from 0 to 100 . Based on this result, each the degree of tinnitus discomfort of each patient was classified as light (0-16), mild (18-36), moderate (38-56), severe (58-76), or catastrophic (78-100).

After the analysis of the two instruments, a statistical analysis of the Spearman correlation coefficient was performed.

For validity, the TFI was correlated with the THI domains for tinnitus severity and was evaluated with Spearman rho. This coefficient ranges from - 1 to 1 -the closer it is to 1 or -1 , the stronger the association; the closer it is to zero, the weaker the relationship between the two variables.

\section{Results}

In the translation stage, the choice of easily understandable words used nationwide was relevant. The items with divergences between translators, modified by consensus of the experts, were:

Question 10 (How often did your tinnitus make it difficult to FALL ASLEEP or MAINTAIN ASLEEP?), in which the term fall asleep was translated as the act of sleeping in one translation and how to remain asleep in another. The term FALL ASLEEP was kept, as it seemed more appropriate to the content of the original version.

Question 15 (Your ability to FOLLOW CONVERSATIONS in a group or meetings?), the term follow conversations was translated as accompanying conversations and how to follow conversations. The term FOLLOW CONVERSATIONS was kept, as it seemed to be more usual.

Question 16 (Your QUIET RESTING ACTIVITIES?) was translated as your quiet activities at rest and as your rest period in silence. We used the term REST ACTIVITIES IN SILENCE, as it seemed to be more appropriate.

The committee of experts mainly discussed item 16 , but maintained the version generated by the consensus.

The Brazilian Portuguese version of the TFI had high reliability, as evidenced by the Cronbach $\alpha(\alpha=0.870)$. For the validation of the TFI, the Spearman correlation test was used, and a strong correlation was found $(p=0.760$ and $p=0.000)$ (-Fig. 1). 


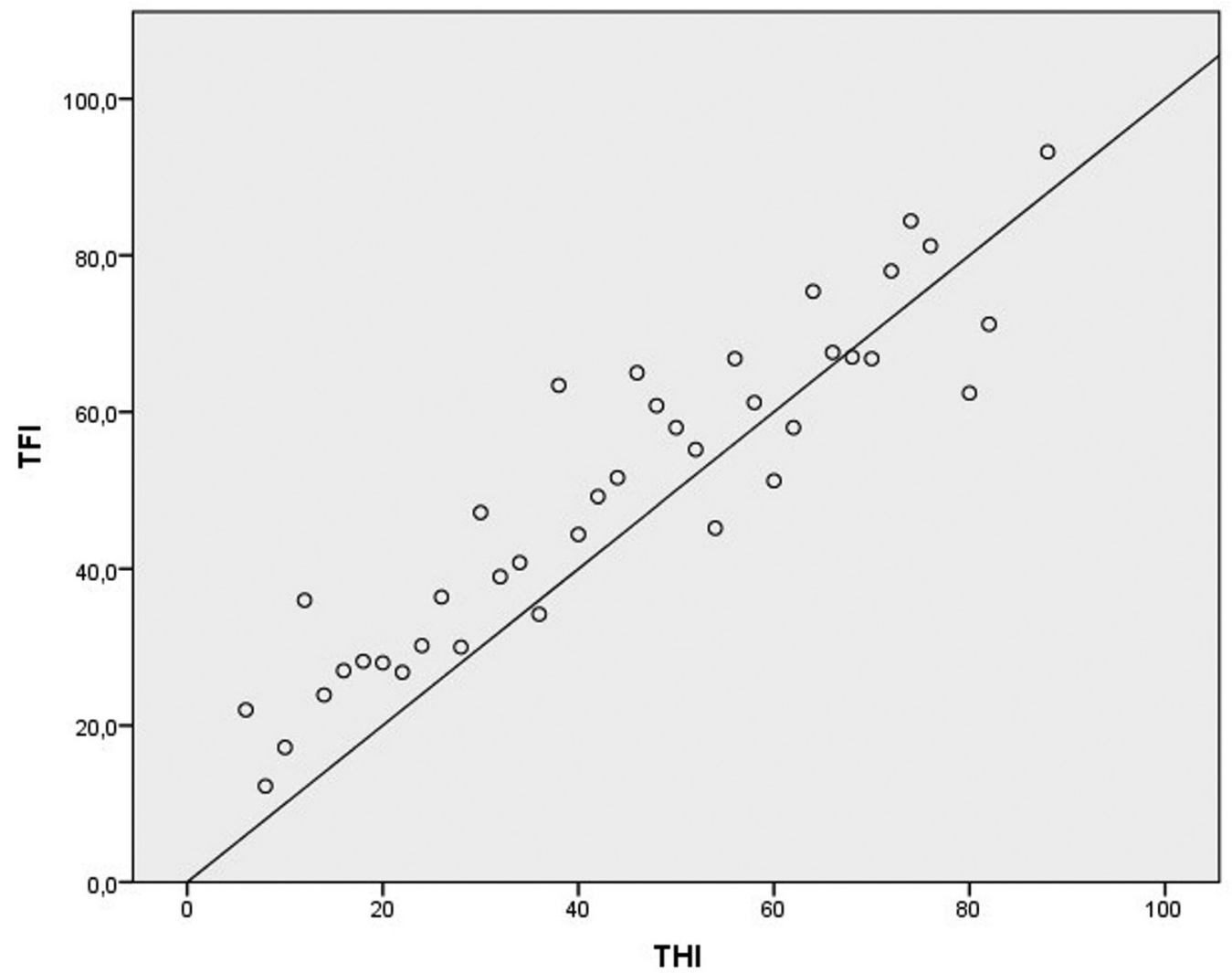

Fig. 1 Correlation between the THI and TFI total scores $(R=0.760 ; p=0.000 ; n=88)$.

Reliability with Cronbach $\alpha=0.870$.

\section{Discussion}

The TFI is a recent questionnaire developed to measure treatment outcomes that is highly responsive to treatment-related change. ${ }^{22}$ In addition, it has a potential focus on identifying factors, through the various domains, which can influence these responses. ${ }^{27}$ Questionnaires are validated in different settings to optimize their use as a standard and should retain most of their original items and internal structure when validated in different populations. ${ }^{30}$

The Brazilian version of the TFI had high reliability and can thus be used as a questionnaire to assess the severity and negative impacts of tinnitus, as well as to measure treatment-related changes. It provides comprehensive coverage of various tinnitus severity domains. ${ }^{20}$

The present study aimed to translate the TFI into Brazilian Portuguese, validate it, and culturally adapt the questionnaire. Therefore, the authors of the present study were careful to evaluate the population in different regions of Brazil. After analyzing the translations and expressions used, a great semantic equivalence to the original TFI was observed, even after the changes made by the experts.

The internal consistency reliability of the Brazilian version of the TFI was high, with a Cronbach $\alpha$ of 0.87 , which is acceptable and comparable to the original, and the Swiss versions - whose Cronbach $\alpha$ were $0.97^{20,31,32}$-, as well as the Polish (0.93), ${ }^{27}$ Chinese (0.94), ${ }^{26}$ and Swedish $(0.95))^{33}$

A strong correlation was found between the THI and the TFI ( $p=0.760)$ - which was to be expected, as the 2 questionnaires are rather similar, with 25 items in different tinnitus domains. The same correlation is also observed in the original ${ }^{20}$ and in other versions. ${ }^{21,27,31-33}$

The Brazilian version of the TFI had high reliability and, therefore, is a questionnaire that can be used to assess the severity and negative impact of tinnitus, as well as to measure treatment-related changes. It provides comprehensive coverage of various tinnitus severity domains. ${ }^{20}$

Finally, the noticed strengths of the TFI are its scope of various tinnitus severity domains, its speed, simple application, and familiarity by the professionals. These can help to identify important changes in tinnitus perception after therapy, including randomized and controlled scientific studies that have a strong impact and scientific evidence in the field. Also, Brazil will be able to compare its results of scientific research with other international centers.

\section{Study Limitation}

The present study has important limitations regarding the sample size and the statistical tests used. Because of the small sample size, factor analysis could not be used reliably; therefore, it is necessary to use a simpler statistical analysis. 


\section{Conclusion}

After the translation, the adaptation of the index, and approval by the author ${ }^{21}$ of the original version, the Brazilian version of the TFI was obtained. The reliability and validity of this version are quite good and comparable with the original version. The Brazilian version of the TFI is, therefore, an option for measuring tinnitus severity and treatment-related changes.

\section{Conflict of Interests}

The authors have no conflict of interests to declare.

\section{References}

1 Weisz N, Hartmann T, Dohrmann K, Schlee W, Norena A. Highfrequency tinnitus without hearing loss does not mean absence of deafferentation. Hear Res 2006;222(1-2):108-114. Doi: 10.1016/ j.heares.2006.09.003

2 Oiticica J, Bittar RSM. Tinnitus prevalence in the city of São Paulo. Braz J Otorhinolaryngol (Engl Ed) 2015;81(02):167-176

3 Hoare DJ, Gander PE, Collins L, Smith S, Hall DA. Management of tinnitus in English NHS audiology departments: an evaluation of current practice. J Eval Clin Pract 2012;18(02):326-334. Doi: 10.1111/j.1365-2753.2010.01566.x

4 Malouff JM, Schutte NS, Zucker LA. Tinnitus-related distress: A review of recent findings. Curr Psychiatry Rep 2011;13(01): 31-36. Doi: 10.1007/s11920-010-0163-1

5 Searchfield GD, Kobayashi K, Sanders M. An adaptation level theory of tinnitus audibility. Front Syst Neurosci 2012;6(46): 1-9. Doi: 10.3389/fnsys.2012.00046

6 Das SK, Wineland A, Kallogjeri D, Piccirillo JF. Cognitive speed as an objective measure of tinnitus. Laryngoscope 2012;122(11): 2533-2538. Doi: 10.1002/lary.23555

7 Fioretti AB, Fusetti M, Eibenstein A. Association between sleep disorders, hyperacusis and tinnitus: evaluation with tinnitus questionnaires. Noise Health 2013;15(63):91-95. Doi: 10.4103/14631741.110287

8 Bertet S, Baskind A, Londero A, Bonfils L, Viaud-Delmon I, Warusfel O. Design and evaluation of tinnitus synthesis methods: from spectral to spatial matching. Am J Otolaryngol 2013;34(02): 121-132. Doi: 10.1016/j.amjoto.2012.09.014

9 Scott B, Lindberg P. Psychological profile and somatic complaints between help-seeking and non-help-seeking tinnitus subjects. Psychosomatics 2000;41(04):347-352. Doi: 10.1176/appi. psy.41.4.347

10 Manning C, Thielman EJ, Grush L, Henry JA. Perception Versus Reaction: Comparison of Tinnitus Psychoacoustic Measures and Tinnitus Functional Index Scores. Am J Audiol 2019;28(1S) 174-180. Doi: 10.1044/2018_aja-ttr17-18-0041

11 Landgrebe M, Azevedo A, Baguley D, et al. Methodological aspects of clinical trials in tinnitus: a proposal for an international standard. J Psychosom Res 2012;73(02):112-121. Doi: 10.1016/ j.jpsychores.2012.05.002

12 Falkenberg ES, Wie OB. Anxiety and depression in tinnitus patients: 5-year follow-up assessment after completion of habituation therapy. Int J Otolaryngol 2012:1-7. Doi: 10.1155/2012/ 375460

13 Barros MBA, Zanchetta LM, Moura EC, Malta DC, et al. Autoavaliação da saúde e fatores associados. Rev. Saúde Pública 2009;43 (Suppl 2):27-37. Doi: 10.1590/S0034-89102009000900005

14 Theme Filha MM, Szwarcwald CL, Souza Junior PR. Measurements of reported morbidity and interrelationships with health dimensions.. Rev Saude Publica 2008;42(01):73-81
15 Paula Erika Alves F, Cunha F, Onishi ET, Branco-Barreiro FC, Ganança FF. Tinnitus handicap inventory: cross-cultural adaptation to Brazilian Portuguese. Pro Fono 2005;17(03):303-310

16 Cima RF, Crombez G, Vlaeyen JW. Catastrophizing and fear of tinnitus predict quality of life in patients with chronic tinnitus. Ear Hear 2011;32(05):634-641. Doi: 10.1097/AUD.0b013e3182 1106dd

17 Lim JJ, Lu PK, Koh DS, Eng SP. Impact of tinnitus as measured by the Tinnitus Handicap Inventory among tinnitus sufferers in Singapore. Singapore Med J 2010;51(07):551-557

18 Salviati M, Macrì F, Terlizzi S, et al. The Tinnitus Handicap Inventory as a screening test for psychiatric comorbidity in patients with tinnitus. Psychosomatics 2013;54(03):248-256. Doi: 10.1016/j.psym.2012.05.007

19 Zeman F, Koller M, Figueiredo R, et al. Tinnitus handicap inventory for evaluating treatment effects: which changes are clinically relevant? Otolaryngol Head Neck Surg 2011;145(02):282-287. Doi: $10.1177 / 0194599811403882$

20 Meikle MB, Henry JA, Griest SE, et al. The tinnitus functional index: development of a new clinical measure for chronic, intrusive tinnitus. Ear Hear 2012;33(02):153-176. Doi: 10.1097/ AUD.0b013e31822f67c0

21 Henry JA, Griest S, Thielman E, McMillan G, Kaelin C, Carlson KF. Tinnitus Functional Index: Development, validation, outcomes research, and clinical application. Hear Res 2016;334:58-64. Doi: 10.1016/j.heares.2015.06.004

22 Rabau S, Wouters K, Van de Heyning P. Validation and translation of the Dutch tinnitus functional index. B-ENT 2014;10(04): 251-258

23 Brüggemann P, Szczepek AJ, Kleinjung T, Ojo M, Mazurek B. [Validation of the German Version of Tinnitus Functional Index (TFI)]. Laryngorhinootologie 2017;96(09):615-619. Doi: 10.1055/s-0042-122342

24 Suzuki N, Oishi N, Ogawa K. Validation of the Japanese version of the tinnitus functional index (TFI). Int J Audiol 2019;58(03): 167-173. Doi: 10.1080/14992027.2018.1534279

25 Fackrell K, Hall DA, Barry JG, Hoare DJ. Psychometric properties of the Tinnitus Functional Index (TFI): Assessment in a UK research volunteer population. Hear Res 2016;335:220-235. Doi: 10.1016/ j.heares.2015.09.009

26 Kam AC, Leung EK, Chan PY, Cheung AP, Tong MC. Cross-cultural adaptation of the Tinnitus Functional Index for measurement of chronic tinnitus in Hong Kong Chinese patients. Hong Kong Med J 2018;24(04, Suppl 4):42-45

27 Wrzosek M, Szymiec E, Klemens W, et al. Polish Translation and Validation of the Tinnitus Handicap Inventory and the Tinnitus Functional Index. Front Psychol 2016;7(1871):1-11. Doi: 10.3389/fpsyg.2016.01871

28 Guillemin F, Bombardier C, Beaton D. Cross-cultural adaptation of health-related quality of life measures: literature review and proposed guidelines. J Clin Epidemiol 1993;46(12):1417-1432. Doi: 10.1016/0895-4356(93)90142-n

29 Hambleton RKPL. Adapting tests for use in multiple languages and cultures. Soc Indic Res 1998;45(19):153-171. Doi: 10.1023/ A:1006941729637

30 Langguth B, Searchfield GD, Biesinger E, et al. History and questionnaires. In, Textbook of tinnitus: Springer; 2011:387-404

31 Peter N, Kleinjung T, Jeker R, Meyer M, Klaghofer R, Weidt S. Tinnitus functional index: validation of the German version for Switzerland. Health Qual Life Outcomes 2017;15(94):1-9

32 Chandra N, Chang K, Lee A, Shekhawat GS, Searchfield GD. Psychometric validity, reliability, and responsiveness of the tinnitus functional index. J Am Acad Audiol 2018;29(07):609-625

33 Hoff M, Kähäri K. A Swedish cross-cultural adaptation and validation of the Tinnitus Functional Index. Int J Audiol 2017;56(04): 277-285 